\title{
INVENTARIO PARTICIPATIVO DE MAMÍFEROS SILVESTRES EN EL EJIDO SAN IGNACIO, MUNICIPIO DE MORELOS, CHIHUAHUA
}

\section{SALVAdor MARTín MEDINA-TORRES, ${ }^{1, *}$ ErÉNdida LuCERo GASTÉLUM-VIZCARRA, ${ }^{2}$ ESTUARdo LARA- PONCE $^{1}$ Y Hugo HuMbERTO PIÑA-RUIZ ${ }^{1}$}

\author{
${ }^{1}$ Cuerpo Académico Desarrollo Sustentable, Universidad Autónoma Indígena de México-Cuidad Universitaria \\ Intercultural. Mochicahui, El Fuerte, Sinaloa, México. CP 81890. Tel +52 (668) 1441052; e-mail: smedinat@gmail.com \\ ${ }^{2}$ Estudiante de Ingeniería Forestal, Universidad Autónoma Indígena de México - Cuidad Universitaria \\ Intercultural. Mochicahui, El Fuerte, Sinaloa, México. CP 81890; e-mail: e_lucerogv@hotmail.com
}

Recibido: 04/09/2014; aceptado: 09/01/2015

\begin{abstract}
Medina-Torres, S. M., Gastélum-Vizcarra, E. L., Lara-Ponce, E. \& Piña-Ruiz, H. H. 2015. Inventario participativo de mamíferos silvestres en el ejido San Ignacio, municipio de Morelos, Chihuahua. Acta Zoológica Mexicana (n. s.), 31(2): 221-233.
\end{abstract}

RESUMEN. Se realizaron cuatro inventarios participativos de mamíferos silvestres mediante el uso de cámaras-trampa en el ejido San Ignacio y sus anexos, municipio de Morelos, Chihuahua, en un área de bosque templado con predominio de pinos, y en menor proporción bosques de encino-pino. Se registraron visitas individuales en video por cada especie en períodos de 24 horas, considerando cuatro temporadas de muestreo, durante las épocas de sequía y lluvias de 2013 y 2014. En total, se obtuvieron 2,027 registros de video, de los cuales 1,456 fueron efectivos para mamíferos silvestres, con un esfuerzo de muestreo de 426 días-trampa. Se registraron 10 especies repartidas entre nueve familias y cinco órdenes, y la mitad de ellas están enlistadas en la Convención sobre el Comercio Internacional de Especies Amenazadas de Fauna y Flora Silvestres (CITES, por sus siglas en inglés). La primavera 2013 fue la temporada con mayor riqueza de especies. Las especies más abundantes fueron el venado cola blanca, el jabalí de collar y el coatí. La mayor abundancia de mamíferos se registró en sitios con bosque de encino-pino. El involucramiento y participación de los ejidatarios en el inventario, ha favorecido la toma de conciencia sobre la importancia de su patrimonio zoocultural, y la necesidad de su conservación. La información generada servirá de base en la elaboración de programas de manejo autogestivos, para la regulación de los aprovechamientos de mamíferos silvestres con fines de subsistencia por los pobladores del ejido.

Palabras clave: Mamíferos terrestres, Sierra Madre Occidental, video-trampeo.

\section{INTRODUCCIÓN}

Los mamíferos silvestres terrestres son uno de los grupos de animales más utilizado por las comunidades indígenas y campesinas de México, y la importancia de su estudio radica en su presencia en diferentes ecosistemas y agroecosistemas, como es el caso del noroeste del país (Cortés-Gregorio et al. 2013). Ecológicamente, estas comunidades humanas mantienen algún tipo de relación con este importante recurso natural, ya que su aprovechamiento es crucial para la satisfacción de las necesidades básicas a través de la caza, al aportar alimento a bajo costo a
Medina-Torres, S. M., Gastélum-Vizcarra, E. L., Lara-Ponce, E. \& Piña-Ruiz, H. H. 2015. Participatory inventory of wild mammals in ejido San Ignacio, municipality of Morelos, Chihuahua. Acta Zoológica Mexicana (n. s.), 31(2): 221-233.

ABSTRACT. Four participatory inventories of wild mammals were carried out by using camera traps in the ejido San Ignacio and its surroundings, municipality of Morelos, Chihuahua, in an area of temperate forest pine dominance, and in less proportion oak-pine forests. Individual visits were recorded on video for each species in periods of 24 hours, considering four sampling periods during the dry and rainy seasons of 2013 and 2014. In total, 2.027 videos were recorded, of which 1,456 were effective in wild mammals in a sampling period of 426 trap-days. 11 species divided among nine families and five orders were recorded. Half of them are listed in the Convention on International Trade in Endangered Species of Wild Fauna and Flora (CITES). The 2013 dry season was the highest on species richness. The most abundant species were white-tailed deer, collared peccary and coati. The greater abundance of mammals was recorded at sites with oak-pine forest. The involvement and participation of landowners in the inventory, has favored the awareness of the importance of their zoocultural heritage and the need for its conservation. The information generated will serve as a basis for the development of community selfmanagement programs for the regulation of harvesting of wild mammals for subsistence purposes by local residents.

Keywords: Terrestrial mammals, Sierra Madre Occidental, videotrapping.

las familias, y proporcionar la principal fuente de proteína animal. Además de lo anterior, los mamíferos silvestres se aprovechan como materias primas para artesanías diversas, y medicinas (Quijano-Hernández \& Calmé 2002; González-Bocanegra et al. 2011; Cortés-Gregorio et al. 2013). Desde una perspectiva sociocultural, no solo significan un recurso tangible objeto de apropiación y uso para satisfacción de necesidades recreativas, culturales o de subsistencia, sino que forman parte del imaginario colectivo en formas intangibles, ya sea dando nombre a lugares, asociados a leyendas, mitos, arte, folclore, o formando parte de la identidad de diversos pueblos (San- 
tos-Fitá et al. 2009; Vargas-Clavijo 2008 y 2009). En un contexto económico, algunos mamíferos terrestres, como el venado cola blanca, han llegado a convertirse en un producto rentable en actividades de turismo cinegético (Medina-Torres 2008; Zamorano de Haro 2009).

Ya se trate de la satisfacción de necesidades alimenticias, económicas o culturales, y ya sea dentro de la legalidad o fuera de ella, la caza de subsistencia es una realidad que sigue practicándose en las diversas regiones del país. Sin embargo, la pérdida de la apropiación cultural de la fauna por los habitantes del medio rural genera una desvalorización del recurso, favorece la extracción excesiva y la comercialización ilegal de especies, sin un control del número de animales cazados, ni un mercado legal para los productos derivados de dichas actividades (QuijanoHernández \& Calmé 2002; González-Bocanegra et al. 2011; Alvarado-Martínez 2012). Esta situación se asocia a la destrucción y fragmentación de los hábitats, la tala y tráfico ilegal de madera (Mathews 2006; Martínez-Ibarra \& Arellano-Montoya 2010), así como a otros fenómenos sociales como el narcotráfico (Astorga 2012). En su conjunto esta compleja problemática afecta y diezma las especies y poblaciones de la fauna silvestre a nivel local y regional. Tal es el caso de las comunidades rurales del Ejido San Ignacio y sus anexos, en el municipio de Morelos, Chihuahua, que se encuentran asentadas en una región de difícil acceso de la Sierra Madre Occidental, y donde el uso de los mamíferos silvestres hasta el presente, se realiza sin regulación alguna. Para garantizar que tales aprovechamientos cumplan con los criterios de sustentabilidad previstos en la normatividad aplicable, se requiere la elaboración de un programa de manejo comunitario, reconocido y supervisados por las autoridades ambientales, y operado en forma responsable por los ejidatarios. Para ello, es necesario hacer el inventario de las especies existentes y su seguimiento, y una forma de lograrlo es mediante el video-trampeo participativo, en donde además de la tecnología, se aprovecha el conocimiento tradicional de los lugareños en torno a las especies y sus indicios. El uso de cámaras-trampa ha venido perfilándose como una alternativa en investigaciones científicas en el mundo sobre tan importante recurso natural. Desde 1990 y hasta 2013 se han publicado en el mundo 71 artículos en los que se utilizaron cámaras- trampa para el estudio de la vida silvestre (Chávez et al. 2013). En Latinoamérica, las cámaras-trampa se han utilizado para realizar estimaciones de la abundancia relativa de distintas especies de fauna (Sáenz-Méndez et al. 2005; Lozano-Rodríguez 2010; Monroy-Vilchis et al. 2011; Aranda et al. 2012; Mesa-Zavala et al. 2012), y constituyen una valiosa herramienta en la realización de inventarios de especies (Aguilar-López et al. 2013; Botello et al. 2008), así como en la gestión de especies cinegéticas, el control de especies o la educación ambiental (Monsalve-Buriticá 2013). Su empleo hace posible el registro de observaciones en tiempo real y de forma remota, con aplicaciones ilimitadas, y evita la necesidad de estar presente para la toma de datos durante períodos considerables de tiempo y bajo condiciones de inseguridad o peligro. Pueden obtenerse tendencias y estimaciones confiables de poblaciones de la fauna silvestre, en especial de las consideradas raras o en peligro de extinción, como es el caso de algunos carnívoros (López-González et al. 2011), y además permite el estudio y seguimiento participativo de las especies a nivel comunitario, ya que el uso de cámaras-trampa ha demostrado ser una estrategia que aumenta notablemente el interés local y regional por la conservación y el adecuado uso de recursos naturales en especial del patrimonio zoo-cultural (Vargas-Clavijo 2009; Botello et al. 2011), pues son los lugareños quienes mejor conocen los sitios y veredas más utilizados por los animales silvestres.

Con base en lo anterior, y con el propósito de generar conocimiento sobre los mamíferos terrestres que habitan los bosques del ejido San Ignacio y sus Anexos, para ser utilizados en futuros programas de manejo comunitario de fauna silvestre, se realizó un estudio de video-trampeo con la participación de algunos ejidatarios de la comunidad de La Fábrica, perteneciente al mencionado ejido. Particularmente se buscó: a) identificar las especies, b) determinar su abundancia en cada tipo de vegetación, y c) evaluar la fiabilidad del inventario.

\section{MATERIAL Y MÉTODOS}

El Ejido San Ignacio y sus Anexos, se ubica entre los $26^{\circ} 24^{\prime} 45.9^{\prime \prime}$ y $26^{\circ} 34^{\prime} 16.5^{\prime \prime}$ de latitud norte, y entre los $107^{\circ} 55^{\prime} 49.9$ ” y 10748’24.3” longitud oeste (Figura 1), con una altitud entre 880 y 2080 msnm, y pertenece a la provincia fisiográfica de la Sierra Madre Occidental. Con base en la cartografía de la información fisiográfica serie I del INEGI (escala 1:1, 000,000), la mayor parte del ejido se ubica en la sub-provincia denominada "Gran Meseta y Cañones Chihuahuenses”, en tanto que una pequeña porción al sur-este pertenece a la sub-provincia Gran Meseta y Cañones Duranguenses”. El sistema de topoformas corresponde al de sierras altas con cañones. De acuerdo a la información del Registro Agrario Nacional, tiene una superficie de 7662.2 ha, cuya vegetación está compuesta de bosques de coníferas (Pinus spp) en diversos grados de asociación con latifoliadas (Quercus spp), sitios perturbados con vegetación secundaria arbustiva y pastizales inducidos, predominando los bosques de pino (Cuadro 1).

En el ejido existen 12 localidades rurales habitadas, con una población total de 390 habitantes (182 hombres 

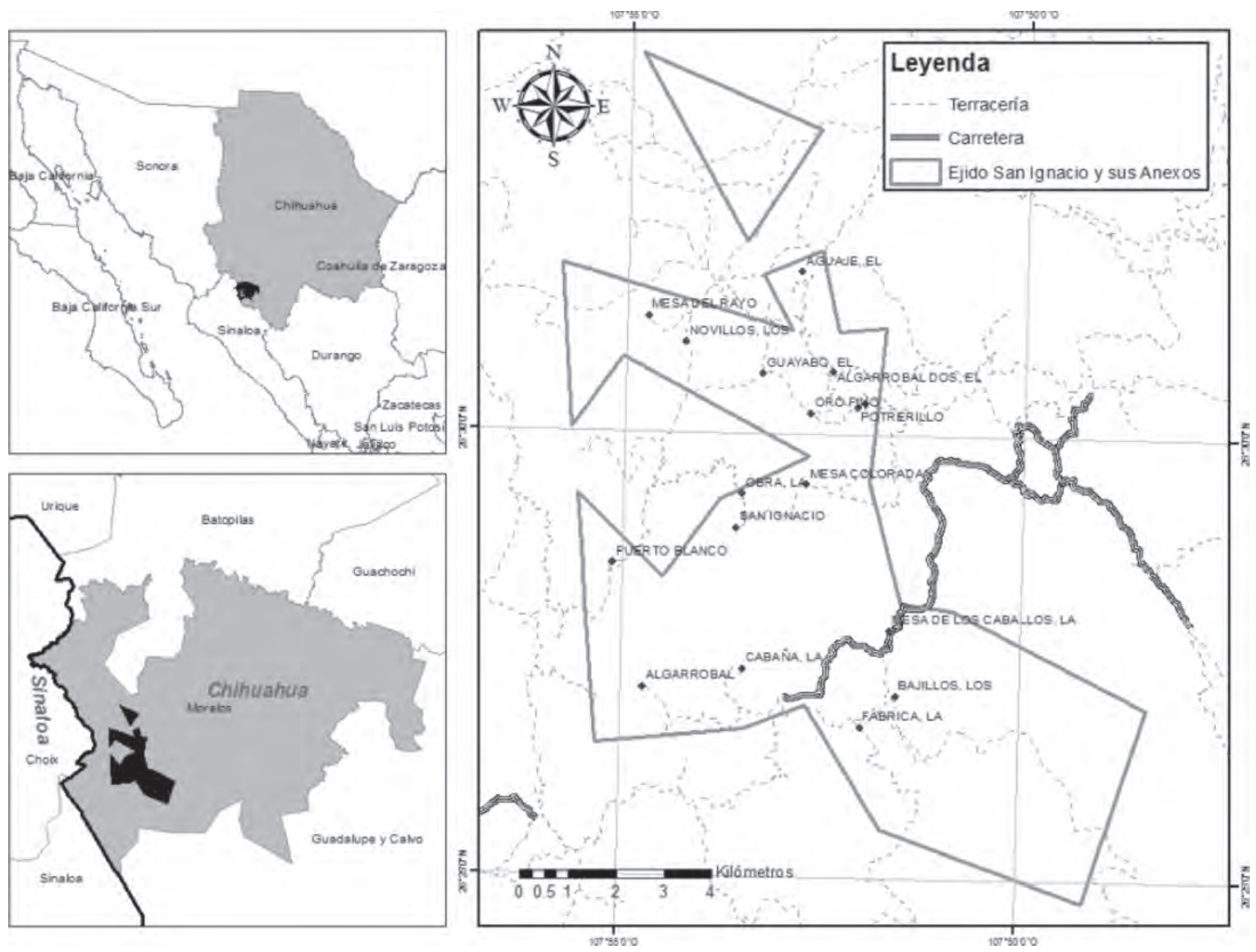

Figura 1. Localización del ejido San Ignacio y sus anexos, municipio de Morelos, Chihuahua.

y 179 mujeres) que viven en 361 hogares (INEGI, 2010). La población indígena en el ejido es de 71 personas de la etnia Rarámuri, de los cuales la mayoría se concentra en el Arrollo del Barranquito, La Fábrica y Los Bajíllos (CDI, 2010). De acuerdo con el Consejo Nacional de Población (CONAPO), la localidad con el más alto grado de marginación es Arrollo del Barranquito, mientras que La Fábrica, Los Bajílos y San Ignacio tienen alta marginación (De la Vega y Téllez, 2010). El ejido se encuentra en la región denominada Baja Tarahumara.

Con el apoyo de las autoridades ejidales e informantes clave de la comunidad de "La Fábrica", en diciembre de 2012 y enero de 2013, se realizó un recorrido previo por el ejido y sus cercanías, para identificar sitios utilizados por la fauna, con condiciones adecuadas de acceso y seguridad. Esto permitió además la capacitación de un grupo de ejidatarios en la instalación y seguimiento de las cámaras-trampa. Las personas participantes en este traba- jo, poseen un amplio conocimiento de la zona, así como de las especies de mamíferos silvestres y sus indicios, en razón de su conocimiento tradicional y experiencia en la caza. Este tiempo fue utilizado como un período de entrenamiento, para probar el funcionamiento de las cámaras con modalidad de fotografía y video, y con distintos períodos de retardo, a fin de elegir la mejor forma para su operación.

Dadas las limitaciones en equipo (solo se contó con cinco cámaras trampa Tasco ${ }^{\circledR}$ ), tiempo para el muestreo (períodos vacacionales de primavera y verano en la institución educativa de los investigadores participantes) y seguridad (el fenómeno del narcotráfico mantiene un clima de inseguridad en la región), y con el fin de capturar la mayor cantidad posible de registros, se decidió considerar cuatro períodos de muestreo: época de secas (abril-mayo de 2013 y 2014), y época de lluvias (julio-agosto de los mismos años). El criterio de selección de las estaciones 
Cuadro 1. Distribución porcentual de los tipos de vegetación en el ejido San Ignacio y sus Anexos.

\begin{tabular}{lc}
\hline Tipo de vegetación & $\%$ \\
\hline Bosque de pino & 50.2 \\
Vegetación secundaria arbustiva de bosque de pino & 20.4 \\
Bosque de encino-pino & 12.5 \\
Bosque de pino-encino & 6.8 \\
Pastizal inducido & 4.8 \\
Vegetación secundaria arbustiva de bosque de encino & 2.4 \\
Vegetación secundaria arbustiva de bosque de encino-pino & 2.1 \\
Vegetación secundaria arbustiva de bosque de pino-encino & 0.8 \\
Total & 100.0 \\
\hline
\end{tabular}

Fuente: elaboración propia con base en la cartografía de uso de suelo y vegetación serie V de INEGI.

de muestreo, consistió en establecer las cámaras-trampa en donde se encontraron evidencias de mamíferos silvestres durante los recorridos previos, como cuerpos de agua, senderos, sitios de alimentación y saladeros naturales (Figura 2).

Se decidió que, si después de una semana una estación no ofrecía resultados, la cámara-trampa se cambiaría a otra ubicación. Cada estación de muestreo fue geo-referenciada con un navegador satelital Garmin ${ }^{\circledR}$ modelo etrex vista, con una proyección UTM y datum WGS-1984, y se registró el tipo de vegetación conforme a la carta de uso de suelo y vegetación serie V de INEGI. Dado que se buscó captar la mayor expresión posible de la diversidad de especies de mamíferos, se decidió que la distancia entre las estaciones fuera de al menos $500 \mathrm{~m}$, que es la recomendada para el caso de especies menores a los 10 kg (López-González et al., 2011). La máxima distancia entre cámaras-trampa fue de 2,677 m. Las cámaras-trampa se programaron para tomar secuencias de video de 15 segundos, con retardo de cinco segundos.

La primera temporada de muestreo (época seca 2013) comprendió cinco estaciones de muestreo. Para la siguiente temporada (lluvias 2013), solo se pudo acceder a tres estaciones de la temporada anterior, por el mal estado de los caminos o las condiciones de inseguridad, por lo que se establecieron cuatro nuevas estaciones, para un total de siete. En la época seca 2014 solo se pudo acceder a tres estaciones utilizadas en los dos muestreos previos, por lo que se establecieron dos nuevas estaciones, y para la época de lluvias de ese año, se visitaron cuatro de las estaciones utilizadas en muestreos anteriores, por lo que se estableció una nueva estación.

El tiempo de operación de las cámaras-trampa varió entre cinco y 38 días. Se revisaron cada semana, ya fuera en vehículo automotor o a caballo, según las condiciones climáticas lo permitieron. Durante la época de sequía
2013, una cámara-trampa falló después de nueve días de operación, en tanto que en esa temporada pero de 2014, ocurrió lo mismo después de cinco días.

Procesado de fotografías e identificación de especies

Las cámaras trampa son sensibles a cualquier tipo de movimiento (viento, sombras, personas, perros, etc.), y se obtuvo un gran número de videos, por lo que se calculó el porcentaje de videos efectivos mediante una adaptación a lo planteado por Lozano-Rodríguez (2010; Ecuación 1):

$$
\% \text { videos efectivos }=\frac{\text { No. de videos efectivos }}{\text { No. de videos totales }}
$$

Donde: videos efectivos $=$ aquellos en los cuales se capturo uno o más individuos de cualquier especie de mamífero silvestre; videos totales $=$ Número total de videos obtenidos, incluyendo aquellos en los que se disparó el sensor por causas diferentes a los mamíferos silvestres.

Las especies fueron identificadas con base en la experiencia y conocimiento de los lugareños participantes, y con el apoyo de una guía de campo (Reid 2006). La información derivada del proceso anterior se integró en una base de datos, que incluyó información del registro obtenido en video, estación de muestreo, tipo de vegetación, fecha, hora, georreferenciación, elevación, especie registrada y número de individuos.

\section{Abundancia por especie}

La abundancia absoluta se calculó para cada especie en cada tipo de vegetación, a partir de los registros individuales de video obtenidos en períodos de 24 horas (un día), ya que se supone que todas las especies tendrían la misma probabilidad de ser registradas por las cámarastrampa (Botello et al. 2008; Mesa-Zavala et al. 2012). Para estimar con mayor precisión la abundancia y evitar en lo posible contar varias veces al mismo individuo, 


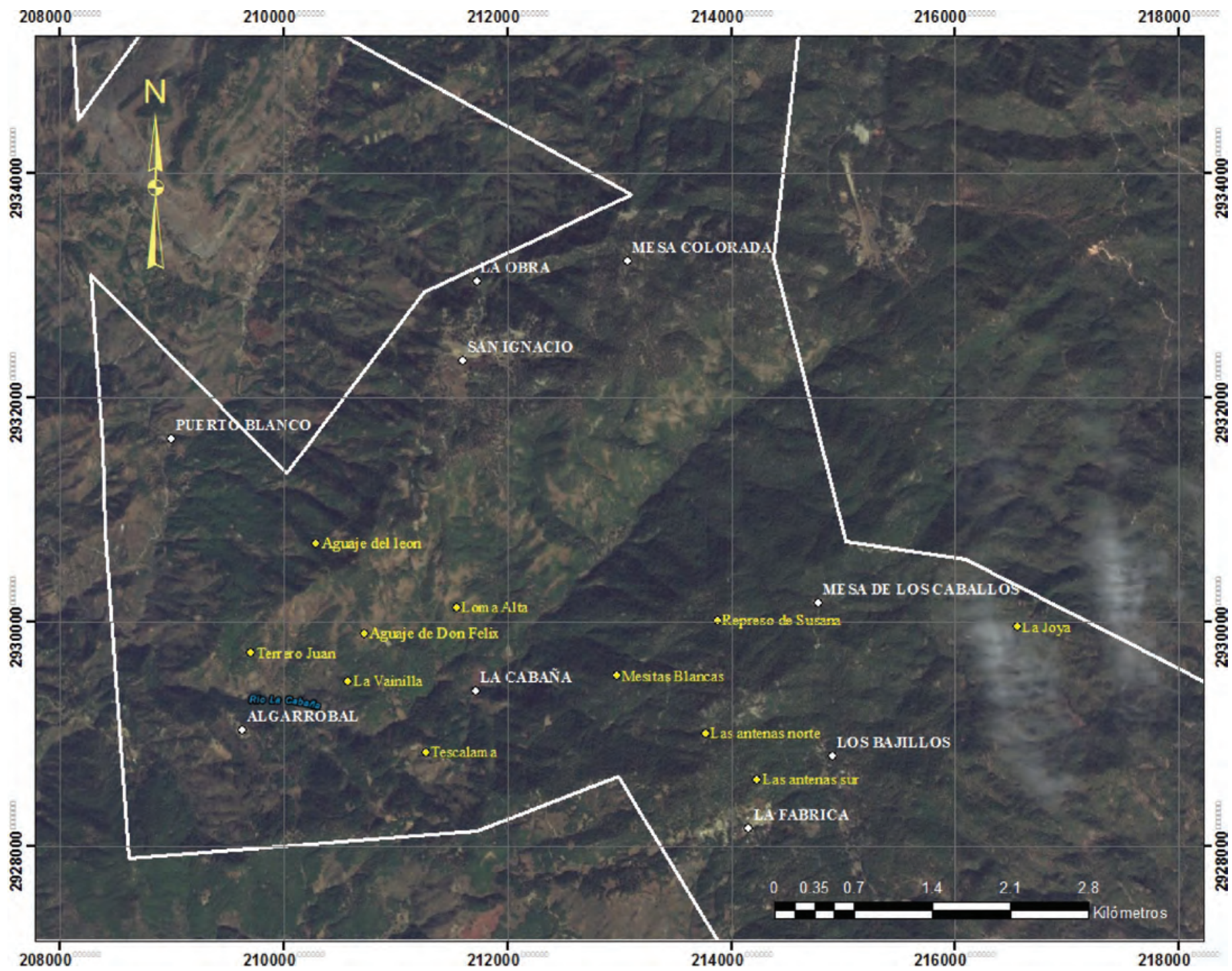

Figura 2. Estaciones de foto-trampeo utilizadas (nombres en altas y bajas) y comunidades rurales (en mayúsculas) en el área de estudio. Las expediciones de video-trampeo partieron de "La Fábrica”.

se consideraron como registros de video independientes los siguientes casos, en una variación a lo propuesto por Monroy-Vilchis et al. (2011): a) videos consecutivos de diferentes individuos, b) videos consecutivos de individuos de la misma especie separados por más de 24 horas (cuando no era posible distinguir si una serie de videos correspondían al mismo individuo en un día se consideraron como un sólo registro), y c) videos no consecutivos de individuos de la misma especie (en caso de especies gregarias como el coatí o el jabalí, cada individuo correspondió a un registro). Con los registros individuales obtenidos, se obtuvo la abundancia relativa.

\section{Evaluación de la fiabilidad de los inventarios}

Considerando todos los registros obtenidos durante el video-trampeo, y para evaluar la fiabilidad de cada inventario, se utilizó el procedimiento descrito por Jiménez-
Valverde y Hortal (2003), el cual consiste en la elaboración de curvas de acumulación de especies, en donde la variable independiente fue el esfuerzo total de muestreo (díastrampa acumulados: la suma de los días-trampa que cada estación permaneció activa; Monroy-Vilchis et al. 2011), y la variable dependiente fue el estimador no paramétrico Jacknife de primer orden (Jack 1), obtenido con el programa EstimateS versión 9.1.0 (Colwell 2013). Posteriormente, cada curva fue ajustada mediante la función de Clench (Soberón y Llorente 1993; Jiménez-Valverde y Hortal 2003; Ecuación 2):

$$
S n=a-n /(1+b-n)
$$

Donde $S n$ = las especies estimadas, $a$ = la tasa de incremento de nuevas especies al comienzo del inventario, $b=$ un parámetro relacionado con la forma de la curva y $n$ = número de unidades de esfuerzo. El ajuste de la función 
se realizó con un modelo de regresión no lineal mediante el algoritomo Simplex \& Quasi-Newton, basado en el procedimiento geométrico para minimizar la loss function utilizando el programa STATISTICA 12.0 (StatSoft, Inc. Tulsa $^{\circledR}$ ). El ajuste de cada modelo se evaluó con base en el coeficiente de determinación $\left(R^{2}\right)$, y el número total predicho de especies de cada curva se obtuvo de su asíntota $(a / b)$. La calidad del inventario se determinó por el cálculo de la pendiente al final de la curva (ecuación 3), y se consideró dicho inventario como completo y fiable, si el valor resultante fue inferior a 0.1 .

$$
\text { Pendiente }=a /(1+b-n)^{2}
$$

Donde $a, b$ y $n$ ya han sido definidos previamente.

El cálculo de la proporción inventariada del total de la fauna en cada temporada de muestreo, se obtuvo por la división del número de especies derivado del estimador Jack 1, por el predicho por la asíntota (Jiménez-Valverde y Hortal 2003; Ecuación 4):

$$
\text { Proporción inventariada }=\operatorname{Sob}_{J_{\text {ack } 1} /\left(\frac{a}{b}\right)}
$$

Donde $S o b_{\text {jacknife } 1}=$ el número promedio de especies dado por el estimador.

Se consideró que a partir de proporciones superiores al $70 \%$, las estimaciones de la riqueza asintótica son estables. Asimismo, se calculó el esfuerzo necesario en díastrampa, para registrar al menos el 75\% de las especies estimadas en el área de estudio (Jiménez-Valverde y Hortal 2003; Ecuación 5):

$$
n_{q}=q /[b \cdot(1-q)]
$$

Donde $n_{q}=$ el número de unidades de esfuerzo necesarias, y $q=0.75$.

\section{RESULTADOS}

\section{Especies registradas y abundancia}

Durante las cuatro temporadas de muestreo, se obtuvieron 2,027 registros en video, de los cuales 1,456 (71.8\%) fueron efectivos para mamíferos silvestres, con un esfuerzo

\begin{tabular}{|c|c|c|c|c|c|c|}
\hline Temporada & Tipo de vegetación & Sitio de muestreo & Videos totales & Videos efectivos & $\%$ efectividad & Días trampa \\
\hline \multirow[t]{5}{*}{ Secas 2013} & Bosque de encino-pino & Aguaje de Don Félix & 175 & 85 & 48.6 & 9 \\
\hline & Bosque de pino & Represo de Susana & 21 & 1 & 4.8 & 25 \\
\hline & & Mesitas Blancas & 9 & 0 & 0.0 & 17 \\
\hline & & Aguaje del León & 59 & 24 & 40.7 & 13 \\
\hline & Totales & & 269 & 110 & 40.9 & 86 \\
\hline \multirow{5}{*}{ Lluvias 2013} & & Loma Alta & 39 & 19 & 48.7 & 26 \\
\hline & & La Vainilla & 13 & 3 & 23.1 & 13 \\
\hline & & Terrero Juan & 59 & 45 & 76.3 & 21 \\
\hline & Bosque de pino & Represo de Susana & 22 & 0 & 0.0 & 36 \\
\hline & & Aguaje del León & 4 & 1 & 25.0 & 18 \\
\hline \multirow{5}{*}{ Secas 2014} & Bosque de pino & Cerro de las antenas Norte & 32 & 0 & 0.00 & 11 \\
\hline & & Cerro de las antenas Sur & 42 & 0 & 0.00 & 5 \\
\hline & & Represo de Susana & 13 & 0 & 0.00 & 11 \\
\hline & & Aguaje del león & 26 & 6 & 23.08 & 11 \\
\hline & Totales & & 296 & 86 & 29.05 & 49 \\
\hline \multirow[t]{5}{*}{ Lluvias 2014} & Bosque de encino-pino & Aguaje de Don Félix & 148 & 137 & 92.57 & 38 \\
\hline & & Terrero Juan & 417 & 408 & 97.84 & 24 \\
\hline & Bosque de pino & Represo de Susana & 5 & 0 & 0.00 & 20 \\
\hline & & Aguaje del león & 6 & 0 & 0.00 & 19 \\
\hline & VSA-Bosque de encino-pino & Tescalama & 35 & 23 & 65.71 & 38 \\
\hline
\end{tabular}
de muestreo de 426 días-trampa (Cuadro 2). Se registra-

Cuadro 2. Efectividad del video-trampeo y registros por tipo de hábitat durante las dos temporadas de muestreo 2013-2014. 
ron 11 especies repartidas entre nueve familias y cinco órdenes. La temporada de sequía 2013 mostró la mayor riqueza de especies. El venado cola blanca (Odocoileus virginianus) apareció en todas las temporadas de muestreo. El jabalí de collar (Pecari tajacu), la ardilla (Sciurus spp), el conejo (Sylvilagus spp) y el coatí (Nasua narica), aparecieron en tres de las temporadas. El ratón (Baiomys spp) y el zorrillo espalda blanca (Conepatus mesoleucus mesoleucus) aparecieron durante dos temporadas, y el resto de las especies solo en una temporada de muestreo (Cuadro 3; Figura 3). Cabe decir que cinco especies están enlistadas en el CITES y ninguna en la NOM-SEMARNAT-059-2010 (DOF, 2010). La especie con mayor abundancia relativa durante tres de las cuatro temporadas de muestreo fue Odocoileus virginianus, seguida de $P e$ cari tajacu y de Nasua narica. Las estaciones ubicadas en bosques de encino fueron las que captaron la mayor abundancia relativa basada en registros individuales, seguidas de las ubicadas en sitios de vegetación secundaria arbustiva de encino-pino, y de aquellas colocadas en sitios de bosque de pino (Figura 4). Durante las lluvias de ambos años, solo se pudo muestrear en una estación ubicada en un bosque secundario de encino-pino con vegetación secundaria arbustiva, y que durante 2013, presentó el 43\% de los registros individuales de venado esa temporada en particular.

\section{Fiabilidad de los inventarios}

El estimador no paramétrico Jacknife de primer orden aplicado a cada temporada de muestreo arrojó resultados contrastantes. En términos generales, los modelos de Clench de las curvas de acumulación de especies de los inventarios de 2013 mostraron un ajuste superior al 99\%, y una pendiente de la curva inferior a 0.1 , lo que indica que dichos inventarios pueden considerarse fiable (Jiménez-Valverde y Hortal 2003). Los inventarios de 2014, aun cuando también mostraron una pendiente inferior a 0.1 , muestran resultados que obligan a tomar con reserva esta aparente fiabilidad (Cuadro 4). Así, el inventario de la época de secas 2014, no alcanzó a cubrir el 70\% de las especies estimadas, lo que sugiere que pudo no haberse

Cuadro 3. Especies registradas, estatus de riesgo y frecuencia de aparición por temporada de muestreo 2013-2014.

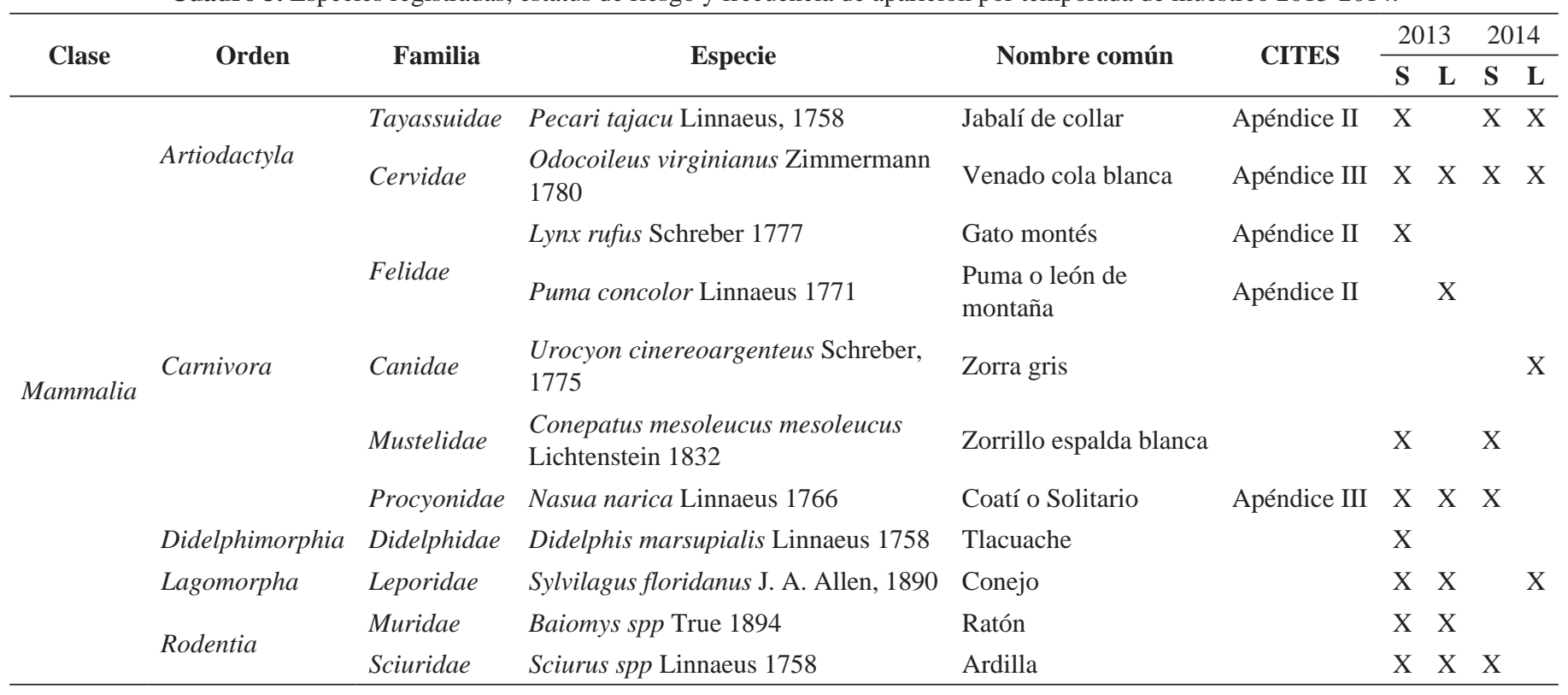

$\mathrm{S}=$ Secas, $\mathrm{L}=$ Lluvias.

Cuadro 4. Modelos de Clench para el estimador Jacknife de primer orden por unidad de esfuerzo para los inventarios de 2013 y 2014.

\begin{tabular}{lcccccccccc}
\hline $\begin{array}{c}\text { Período de } \\
\text { muestreo }\end{array}$ & $\mathbf{n}$ & $\begin{array}{c}\text { Especies } \\
\text { registradas }\end{array}$ & $\mathbf{R}^{2}$ & $\begin{array}{c}\text { Promedio de } \\
\text { spp estimadas } \\
\text { (Jack 1) }\end{array}$ & a & b & $\begin{array}{c}\text { Spred } \\
\mathbf{( a / b )}\end{array}$ & $\begin{array}{c}\text { Pendiente } \\
\text { de la curva }\end{array}$ & $\begin{array}{c}\text { \% de las especies } \\
\text { estimadas }\end{array}$ & $\begin{array}{c}\text { Días-trampa para } \\
\text { 75\% de las spp }\end{array}$ \\
\hline Primavera 2013 & 86 & 9 & 0.995 & 11.97 & 0.694 & 0.045 & 16 & 0.030 & 76.86 & 67 \\
Verano 2013 & 152 & 6 & 0.991 & 7.99 & 0.275 & 0.028 & 10 & 0.010 & 81.32 & 60.77 \\
Primavera 2014 & 49 & 3 & 0.981 & 3.98 & 0.254 & 0.039 & 7 & 0.030 & 77 \\
Verano 2014 & 139 & 4 & 0.928 & 5.99 & 0.143 & 0.020 & 7 & 0.010 & 82.82 & 152 \\
\hline
\end{tabular}

Spred = especies predichas (asíntota de la curva). 

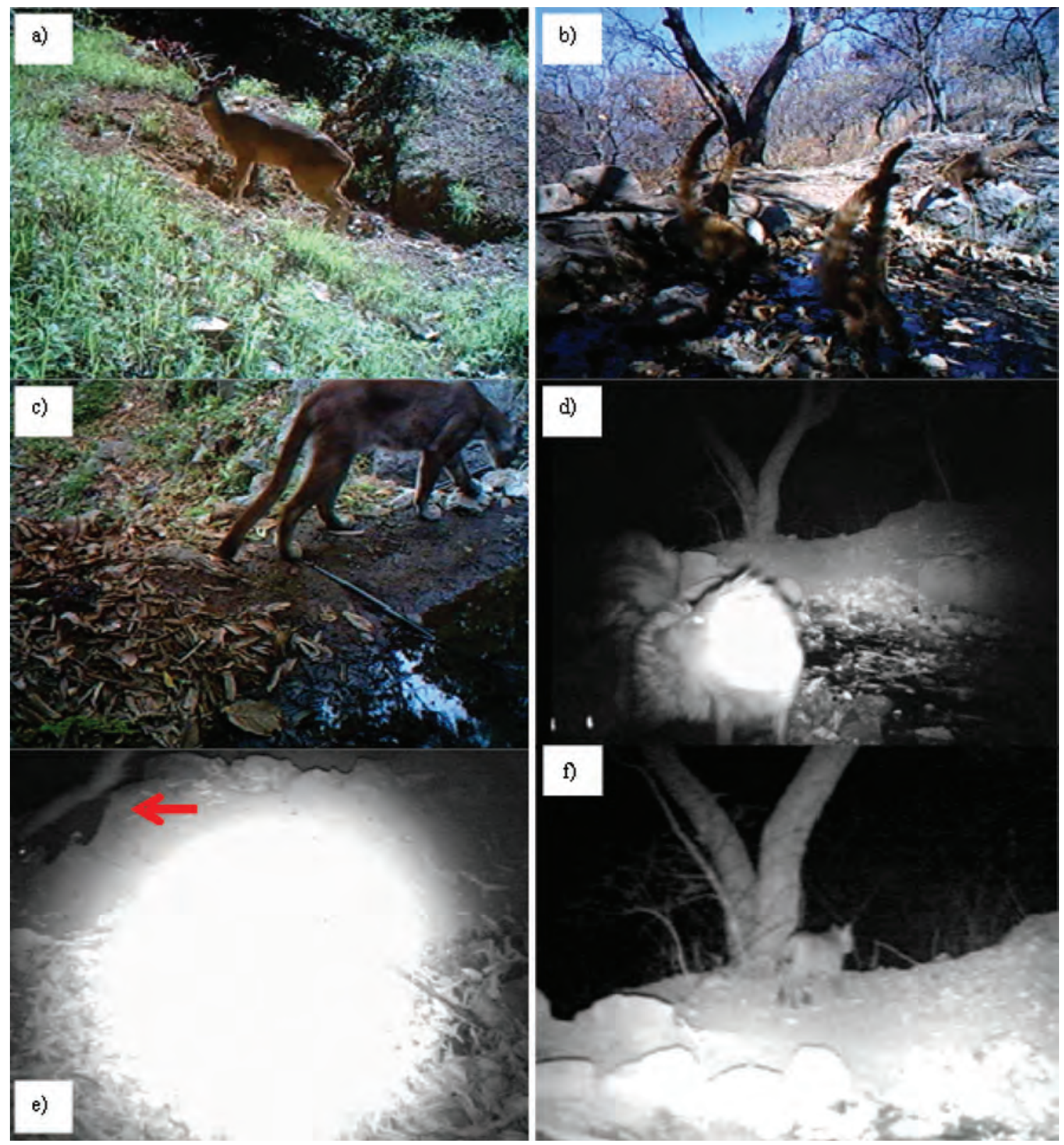

Figura 3. Algunas de las especies registradas: a) venado cola blanca, b) coatí, c) puma, d) jabalí de collar, e) zorrillo de espalda blanca, y f) gato montés.

alcanzado la asíntota de la curva, mientras que el modelo de Clench para el inventario de lluvias de ese mismo año, presentó el menor ajuste (inferior al 95\%), e incluso la curva resultante tiende menos hacia la asíntota (Figura 5). De hecho, los inventarios de 2014 presentaron el mismo número estimado de especies, siendo menor a lo estimado durante 2013, posiblemente en respuesta a una disminución en el esfuerzo de muestreo.

\section{DISCUSIÓN}

Una de las contribuciones de este trabajo ha sido introducir la innovación tecnológica del foto-trampeo en la mo- dalidad de video, como herramienta en la realización de inventarios participativos de fauna silvestre a nivel comunitario. La video-filmación en estudios de fauna silvestre ha utilizado desde mini-cámaras alámbricas de video para circuito cerrado de televisión con sensores de movimiento (Faller-Menéndez et al. 2007), hasta cámaras-trampa que funcionan con pilas e incluso con paneles solares, con capacidad de tomar videos breves de 15 a 60 segundos y con tiempos de retardo cortos, desde unos cuantos segundos a cinco minutos (Delgado et al. 2011; Perea García-Calvo et al. 2009; Harmsen et al. 2010; Santacruz-Salazar 2012), lo que permite capturar con más eficiencia, no solo al ejemplar en sí, sino las actividades que realiza y su duración. 

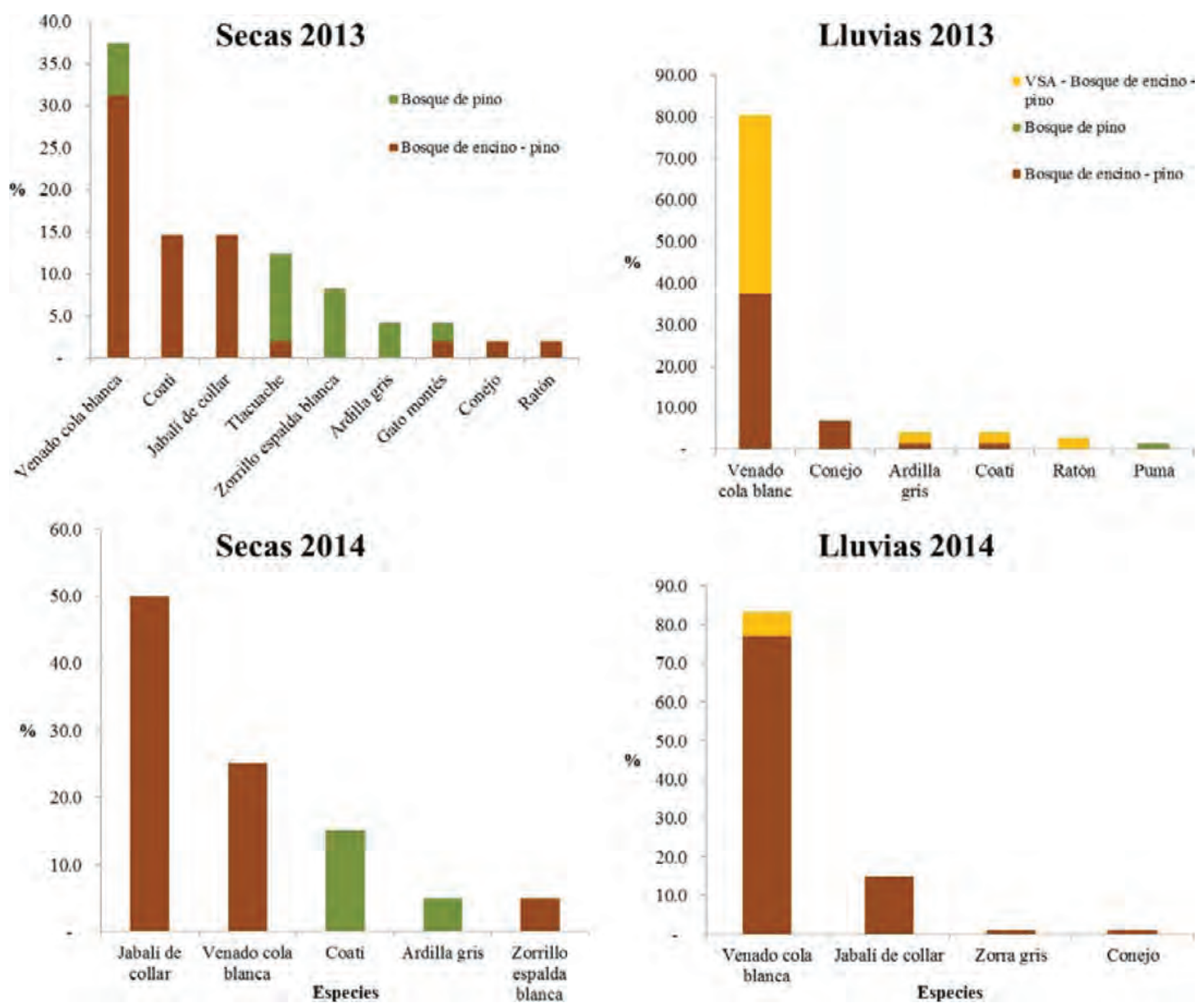

Figura 4. Abundancia relativa por especie y tipo de vegetación durante las dos temporadas de muestreo 2013-2014. El puma fue nuevo registro en las lluvias de 2013, y la zorra gris lo fue en 2014.

Otra de las ventajas que ofrece el uso de cámarastrampa en la realización de inventarios de fauna silvestre, particularmente en la descripción de grupos de fauna, como los mamíferos, es que permite utilizar las curvas de acumulación de especies como un medio para evaluar su fiabilidad y posibilitar su comparación, además de hacer factible una mejor planificación del trabajo de muestreo, al estimar el esfuerzo requerido para conseguir inventarios fiables. Por ejemplo, y con base en el modelo de Clench para este trabajo, si se hubieran incrementado los días trampa en cuatro unidades durante la época seca de 2013, se hubiera alcanzado el $80 \%$ de la riqueza estimada.

Puede observarse que la época seca de 2013 fue la que arrojó el mayor número de especies, debido a la gran afluencia de especies en cuerpos de agua o áreas con alimento. En todos los casos, salvo en el inventario de la época de sequía 2014, el porcentaje de las especies estimadas fue superior al $70 \%$, por lo que cabría esperar que en cada caso, se haya alcanzado la asíntota. En contraste, en el inventario mencionado, apenas si se alcanzó poco más del $60 \%$ de las especies estimadas, lo que indudablemente pudo deberse al bajo esfuerzo de muestreo, ya que en esa época, las condiciones de inseguridad no hicieron recomendable extender más tiempo el video-trampeo, situación que continuó hasta las lluvias de 2014. No obstante, y dado el ajuste de cada modelo y la pendiente de la asíntota, sería factible emplear el valor de ésta como predictor de la riqueza de especies, en lugar del número 


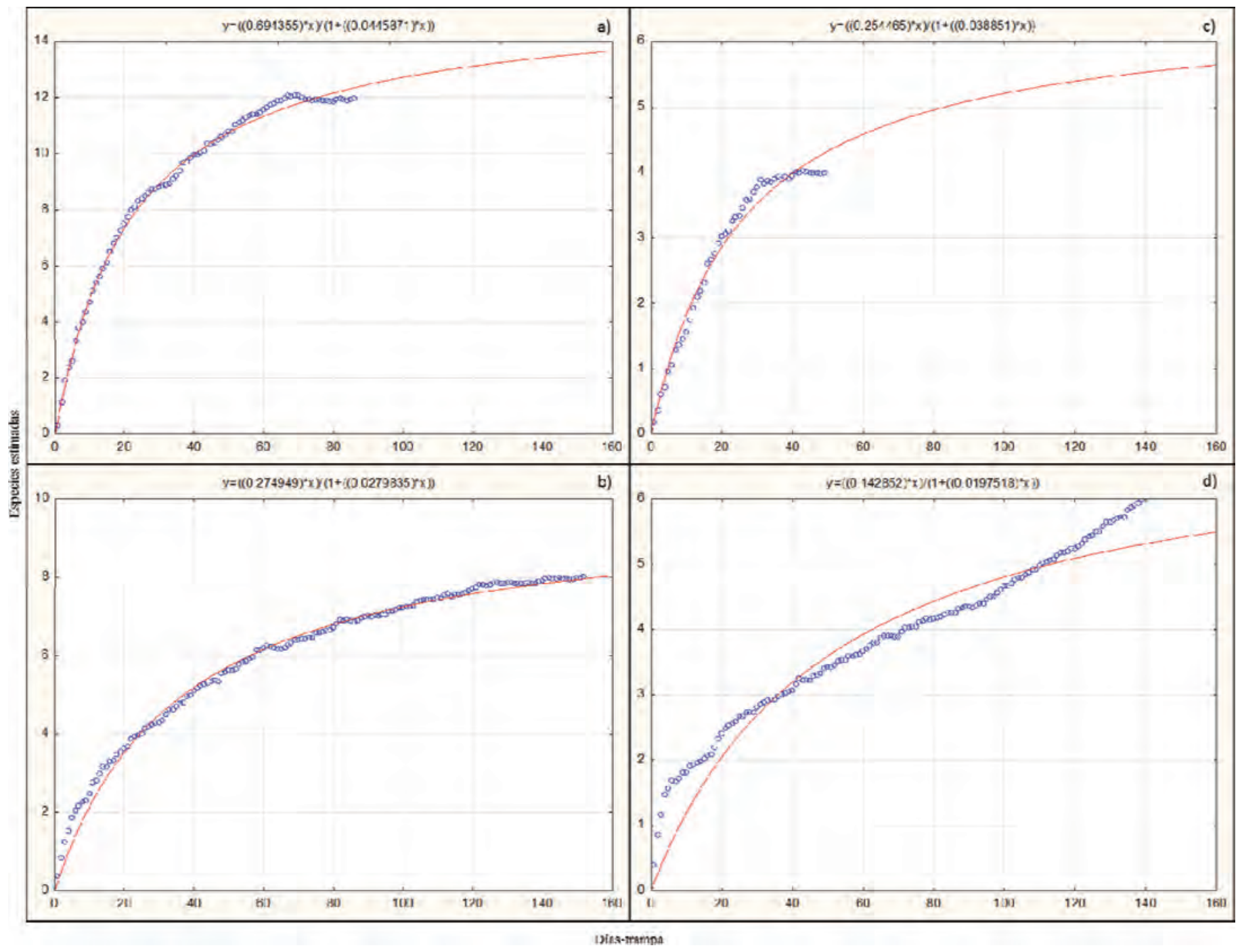

Figura 5. Curvas de acumulación de mamíferos silvestres ajustadas a 160 días-trampa: a) época de secas 2013, b) época de lluvias 2013, c) época de secas 2014, d) época de lluvias 2014. Los puntos corresponden a la riqueza promedio acumulada de mamíferos silvestres del estimador Jack 1, y las líneas indican la curva de especies predicha por el modelo de Clench.

observado de especies (Jiménez-Valverde y Hortal 2003). Considérese que en los cuatro inventarios, se registró un total de 11 especies, lo que significa que se ha logrado registrar el $68.8 \%$ de las especies predichas durante el inventario de la época de sequía 2013.

Estos resultados pueden servir de base para futuros estudios en la zona y detectar fluctuaciones en la riqueza de especies, ya sea por cambios en el hábitat, o presiones antrópicas sobre sus poblaciones, siendo además posible extrapolar el número de especies observado para estimar el total de especies que estarían presentes en una zona más grande (Soberón \& Llorente 1993; Jiménez-Valverde \& Hortal 2003). Botello et al. (2008) encontraron que la máxima riqueza de especies se presentó durante la primavera al utilizar combinaciones de foto-trampas, cepos y estaciones olfativas, y concluyeron que el foto-trampeo fue el método que mejor estimó la riqueza, en comparación con los otros métodos, cuyas curvas no alcanzaron la asíntota. Esto mismo parece haber sucedido en los muestreos de este trabajo, ya que al obtener pendiente de la asíntota inferiores a 0.1 , el inventario con cámaras-trampa si fue confiable, aunque sería posible alcanzar mejores estimaciones al instalar más equipos, con lo cual se incrementaría el esfuerzo de muestreo.

Otro parámetro de interés en un inventario de fauna es la abundancia relativa, ya que permite realizar comparaciones a nivel temporal y espacial (Botello et al. 2008). Aranda et al. (2012), en un foto-trampeo efectuado entre febrero de 2008 y agosto de 2009, encontraron que la especie más abundante en un bosque mesófilo de montaña fue el venado cola blanca (47.5\%), ubicándose entre los valores documentados en este trabajo para las tempora- 
das secas de 2013 (37.5\%) y 2014 (25.0\%) y las lluvias de ambos años (80.6 y 83.3\% respectivamente). Por su parte, Monroy-Vilchis et al. (2011), en un foto-trampeo con mayor duración (diciembre 2003 a mayo 2006), documentaron que esta misma especie se contó entre las más abundantes, solo por debajo del coatí, el conejo, el puma y la zorra gris, en la Reserva Natural Sierra Nanchititla, en el estado de México, donde el hábitat predominante es la selva baja caducifolia, y en menor proporción el bosque de latifoliadas (encino) y bosques de pino-encino, aunque estos autores no especificaron en qué tipo de hábitat el venado fue más abundante.

Las otras especies más abundantes registradas en este trabajo durante la época seca de 2013 fueron el coatí (Nasua narica) y el jabalí de collar (Pecari tajacu; 14.6\% respectivamente), en tanto que para las lluvias de ese año, fueron el conejo (Sylvilagus spp) y la ardilla (Sciurus spp; 6.9 y 4.2 respectivamente). Cabe decir que una especie de conejo (Sylvilagus floridanus), que posiblemente corresponda con la que existe en el ejido San Ignacio y sus Anexos, fue la de mayor abundancia en el bosque mesófilo de montaña de Manantlán (Aranda et al. 2012).

Las cifras del año 2014 presentaron fluctuaciones en relación a las respectivas épocas de muestreo de 2013. Así, aparentemente la abundancia relativa del venado cola blanca durante la sequía de 2014 muestra una disminución importante, que pudiera deberse a un incremento en su caza, aunque el bajo esfuerzo de muestreo en esa temporada pudo causar este resultado. Sin embargo, los coatíes y jabalíes continúan apareciendo entre las tres especies más abundantes durante la época seca.

Si se considera el período de secas y el bosque de encino-pino, el jabalí de collar fue la especie más abundante (junto con el coatí) solo por debajo del venado cola blanca, coincidiendo por lo consignado por Monroy-Vilchis et al. (2011). El jabalí y el coatí, fueron las dos especies de mamíferos de tipo gregario que pudieron ser registradas en el área de estudio; a diferencia de este trabajo, el coatí resultó ser la especie más abundante en selva baja caducifolia Monroy-Vilchis et al. (2011), en tanto que se contó entre las menos abundantes en el bosque mesófilo de montaña (Aranda et al. 2012).

A semejanza de este trabajo (2.1\% en bosque de encino-pino y bosque de pino respectivamente), el gato montés fue uno de los felinos silvestres menos abundantes (1.0\%) documentados por Botello et al. (2008) en un bosque de pino-encino de la Sierra Madre de Oaxaca, y que solo pudieron registrar utilizando cepos y estaciones olfativas, y no con foto-trampeo. Coincidiendo con estos autores, Mesa-Zavala et al. (2012) encontraron que el gato montés fue de las especies menos abundantes en un hábitat semiárido de Baja California Sur durante marzo a octubre de 2017, ya que solo pudieron obtener un registro en uno de cuatro sitios de muestreo consistentes en cuerpos de agua superficiales.

El caso del puma en este trabajo merece especial atención; solo se logró obtener un registro de esta especie, lo que la sitúa en el valor más bajo de abundancia relativa de la época de lluvias 2013 (1.4\%), contrastando con lo consignado por Monroy-Vilchis et al. (2011), quienes encontraron que el puma fue la cuarta especie con mayor índice de abundancia relativa y por encima del venado, en tanto que Aranda et al. (2012) documentaron que este felino fue la especie más abundante (19.2\%), solo por debajo del venado. Lo registrado por los primeros autores en el estado de México plantea una pregunta inquietante, ya que el puma aparece con mayor abundancia relativa por encima de sus presas naturales (venados y jabalíes), lo que lleva a pensar en algún posible desequilibrio en la mastofauna local. En cambio, el único registro que se obtuvo de puma en este trabajo, correspondió a un cuerpo de agua (llamado por los lugareños como el "aguaje del león”), con 18 días de foto-trampeo durante el verano, y sin ninguna otra especie, cuando ese mismo sitio fue muestreado durante la primavera anterior por 13 días, registrando cinco especies, entre las que se encontró el venado cola blanca. Este mismo sitio, fue muestreado en el año 2014, sin que se obtuvieran resultados. Esto hace pensar que la presencia del puma en ese sitio fue la causa de que las poblaciones presa se desplazaran a otros entornos, fuera del territorio de caza del felino. En este sentido, la baja abundancia relativa de felinos silvestres, se corresponde con una abundancia mayor de especies que pueden ser sus presas, lo que sugiere una comunidad mastofaunística en equilibrio.

Una de las mayores contribuciones que este trabajo hizo, fue hacia la toma de conciencia de los ejidatarios de San Ignacio y sus anexos. El reconocer en video las especies que habitan sus tierras, les ha hecho valorar sus recursos faunísticos, ya que han comenzado a involucrarse en el cuidado de su patrimonio zoocultural (Vargas-Clavijo 2008 y 2009), frente a la caza desmedida por avecindados procedentes de otras regiones, particularmente a consecuencia del fenómeno del narcotráfico. Durante las experiencias generadas a lo largo de este estudio, pudieron recabarse valiosos testimonios sobre la fauna silvestre que ya están extintas. Por ejemplo, antes los habitantes del lugar “...tenían mucho miedo a los animales grandes (osos, lobos y leones) y que hacían campañas para buscarlos y matarlos, y así fue como acabaron con estos animales que no dejaban vivir a gusto a la gente...”. Este mismo testimonio da cuenta sobre dos especies de felinos que la gente reconocía, ya que más adelante se cuenta sobre la creencia de que “... el león colorado es amigo de la gente 
que porque muchas veces se supo que peleaba con el león pardo para impedir que se comiera a la gente que andaba de noche en el monte.... De todos modos lo mataban por sí o por no.” (José M. Peña, comunicación personal). Testimonios como este, ponen en evidencia la necesidad de concientizar a los ejidatarios sobre el importante papel de los depredadores en la regulación de las especies presa (venados y jabalíes). La importancia de algunas especies documentadas en este trabajo por su uso alimentario es plenamente reconocida por los ejidatarios. Por ejemplo, la carne del venado es considerada de las mejores, aunque hoy día "... ya no es posible tener siempre carne de venado en la casa porque ya no encuentras venados donde quiera como antes..." (Remedios Peña, comunicación personal). Otra especie es el jabalí (conocido localmente como "cochi”), que los lugareños consideran que “...tienen muy buena carne..." así como otras especies que también se acostumbra comer en la sierra, como los “cholugos” (denominación local para el coatí), apreciados por su carne, aunque también se les considera dañinos en las milpas y por tanto su caza es doblemente justificada (Abel Chaparro, comunicación personal); y el armadillo (Dasypus novemcinctus; Ramón Gil, comunicación personal), especie que no pudo ser registrada durante este inventario.

\section{CONCLUSIONES}

Una de las mayores contribuciones que este trabajo hizo, puede resumirse en dos grandes puntos: por un lado se encuentra la importancia del uso de cámaras-trampa en modalidad de video en el ecosistema templado y serrano de una región de difícil acceso en lo ambiental y lo social, para detectar la riqueza y abundancia de especies de fauna que los lugareños aprovechan, y cuyo conocimiento es la base para cualquier programa de conservación y aprovechamiento sostenible a futuro. Con los resultados de este trabajo, se pueden planificar futuros inventarios con más fiabilidad y precisión, que permitirán el seguimiento de las especies y sus poblaciones a través del tiempo.

Por el otro, se encuentra la percepción social de la gente local (que pervive en su memoria histórica) sobre las especies de la mastofauna que ellos aprovechan y sus implicaciones, al generar con su participación (como producto de este trabajo), una sensibilización para el mejor aprovechamiento de los mismos con un enfoque de conservación in situ.

AGRADECIMIENTOS. Los autores agradecen al PROMEP por los apoyos otorgados para la adquisición de los equipos de foto-trampeo utilizados en esta investigación. Se agradece en particular al Comisariado Ejidal de San Ignacio y sus anexos, así como al Comisario de Policía, y a los ejidatarios participantes y residentes de "La Fábrica" y otras comunidades del área de estudio, por las facilidades otorgadas para la realización de la presente investigación.

\section{LITERATURA CITADA}

Aguilar-López, M., Rojas-Martínez, A. E., Cornejo-Latorre, C., Vite-Silva, V. D., \& Ruano-Escalante, Y. R. 2013. Lista taxonómica y estructura del ensamblaje de los mamíferos terrestres del municipio de Tlanchinol, Hidalgo, México. Mastozoología neotropical, 20(2): 229-242.

Alvarado-Martínez, I. 2012. Delincuencia organizada ambiental en México, una nueva manifestación criminal del tráfico de especies. Rev. Crim., 54(1):283-311.

Aranda, M., Botello, F., \& López-de Buen, L. 2012. Diversidad y datos reproductivos de mamíferos medianos y grandes en el bosque mesófilo de montaña de la Reserva de la Biosfera Sierra de Manantlán, Jalisco-Colima, México. Revista mexicana de biodiversidad, 83(3):778-784.

Astorga, L. 2012. El siglo de las drogas. El narcotráfico, del porfiriato al Nuevo Milenio. México, Editorial Grijalbo-Proceso, 2012, pp. 127-165.

Botello, F., Sánchez-Cordero, V., \& González, G. 2008. Diversidad de carnívoros en Santa Catarina Ixtepeji, Sierra Madre de Oaxaca, México. En: Lorenzo E., Espinosa E. y Ortega, J. (Coordinadores). Avances en el estudio de los mamíferos de México II. 335-354 pp.

Botello, F., E. Villaseñor, M. Aranda, G. Magaña-Cota y L. Guevara. 2011. Guía de Campo para el Monitoreo Participativo de Vertebrados Utilizando Rastros y Fototrampas. Reserva de la Biosfera de Tehuacán-Cuicatlán, Oaxaca, México. Primera edición. Conservación Biológica y Desarrollo Social A.C. México, D. F. $61 \mathrm{p}$.

CDI. 2010. Catálogo de localidades indígenas 2010. Recuperado de: http://www.cdi.gob.mx/index.php?option=com_content\&view=a rticle\&id=2578.

Chávez, C., A de la Torre, H. Bárcenas, R.A. Medellín, H. Zarza \& G. Ceballos. 2013. Manual de fototrampeo para estudio de fauna silvestre. El jaguar en México como estudio de caso. Alianza WWF-Telcel, Universidad Nacional Autónoma de México, México. $103 \mathrm{p}$.

Colwell, R. K. 2013. EstimateS: Statistical estimation of species richness and shared species from samples. Version 9. Persistent URL $<$ purl.oclc.org/estimates>.

Cortés-Gregorio, I., E. Pascual-Ramos, S. M. Medina-Torres, E. A. Sandoval-Forero, E. Lara-Ponce, H. H. Piña-Ruíz, R. Martínez-Ruíz \& G. E. Rojo-Martínez. 2013. Etnozoología del pueblo Mayo-Yoreme en el norte de Sinaloa: uso de vertebrados silvestres. Revista de Agricultura, Sociedad y Desarrollo. Colegio de Postgraduados. 10(3): 335-358.

De la Vega, S., \& Téllez, Y. 2012. Índice de marginación por localidad 2010. Consejo Nacional de Población.

Delgado-V, C. A., Árias-Alzate, A., Botero, S., \& Sánchez-Londoño, J. D. 2011. Behaviour of the Tayra Eira barbara near Medellín, Colombia: preliminary data from a video-capturing survey. Small Carnivore Conservation, 44:19-21.

DOF. 2010. NORMA Oficial Mexicana NOM-059-SEMARNAT2010, Protección ambiental-Especies nativas de México de flora y fauna silvestres-Categorías de riesgo y especificaciones para su inclusión, exclusión o cambio-Lista de especies en riesgo. Diario Oficial de la Federación. 30 de diciembre de 2012.

Faller-Menéndez, J. C., Lago-Torres, L. A., Hernández-Cardona, A., Calleja-Alvarado, M., González, G. C., Tovar, C. C., \& 
Johnson, S. 2007. La video-filmación como técnica de estudio de mamíferos silvestres: un ejemplo de jaguares en el noreste de la Península de Yucatán. Revista Mexicana de Mastozoología (Nueva Época), 11(1):47-56.

González-Bocanegra K., Romero-Berny E. I., Escobar-Ocampo M. C. y García-Del Valle Y. 2011. Aprovechamiento de fauna silvestre por comunidades rurales en los humedales de CatazajáLa Libertad, Chiapas, México. Ra Ximhai (7)2: 219-230.

Harmsen, B. J., Foster, R. J., Gutierrez, S. M., Marin, S. Y., \& Doncaster, C. P. 2010. Scrape-marking behavior of jaguars (Panthera onca) and pumas (Puma concolor). Journal of Mammalogy, 91(5):1225-1234.

INEGI. 2010. Censo de población y vivienda 2010. Instituto Nacional de Estadística y Geografía. Recuperado de: http://www.inegi.org. $\mathrm{mx} /$ sistemas/consulta_resultados/iter2010.aspx? c=27329\&s=est

Jiménez-Valverde, A., \& Hortal, J. 2003. Las curvas de acumulación de especies y la necesidad de evaluar la calidad de los inventarios biológicos. Revista Ibérica de Aracnología, 8:151-161.

López-González C., C. E. Gutiérrez-González y N. E. Lara-Díaz. 2011. Carnívoros: inventario y monitoreo. En: Gallina-Tessaro S. y López-González C. (Ed). Manual de técnicas para el estudio de la fauna. Volumen I. Instituto de Ecología A.C. y Universidad Autónoma de Querétaro. México. 133-160 pp.

Lozano-Rodríguez, L. A. 2010. Abundancia relativa y distribución de mamíferos medianos y grandes en dos coberturas vegetales en el santuario de fauna y flora Otún Quimbaya mediante el uso de cámaras trampa. Tesis de Licenciatura. Pontificia Universidad Javeriana. Facultad de Ciencias. Carrera de Biología. Bogotá, Colombia. 43 pp.

Martínez-Ibarra, J. A. \& R. E. Arellano-Montoya. 2010. Uso comunitario de los recursos naturales en el Ejido Lagunillas, Jalisco, México. Ambiente y Desarrollo. 14(26):95-109.

Mathews, A. S. 2006. Ignorancia, conocimiento y poder. El corte de la madera, el tráfico ilegal y las políticas forestales en México. Desacatos, 21:135-160.

Medina-Torres, S. M. 2008. El venado cola blanca (Odocoileus virginianus couesi) y su hábitat en la Sierra del Laurel, Aguascalientes. Tesis de doctorado. Colegio de Postgraduados Campus Montecillo. Programa de postgrado en Botánica. Montecillo, Texcoco, Edo. de México. 264 p.

Mesa-Zavala, E., Álvarez-Cárdenas, S., Galina-Tessaro, P., Troyo-Diéguez, E., \& Guerrero-Cárdenas, I. 2012. Vertebrados terrestres registrados mediante foto-trampeo en arroyos estacionales y cañadas con agua superficial en un hábitat semiárido de Baja California Sur, México. Revista mexicana de biodiversidad, 83(1):235-245.
Monroy-Vilchis, O., Zarco-González, M. M., Rodríguez-Soto, C., Soria-Díaz, L., \& Urios, V. (2011). Fototrampeo de mamíferos en la Sierra Nanchititla, México: abundancia relativa y patrón de actividad. Revista de Biología Tropical, 59(1), 373-383.

Monsalve-Buriticá, S. 2013. Metodologías para la Colecta de Muestras en Fauna Silvestre in situ. En: Memorias de la Conferencia Interna en Medicina y Aprovechamiento de Fauna Silvestre, Exótica y no Convencional, 9(2):46-57.

Perea García-Calvo, R., \& Ayanz, S. M. 2009. Dispersión secundaria de semillas de robles (Quercus petraea y Quercus pyrenaica) en el Hayedo de Montejo (Madrid). En: Congresos Forestales.

Quijano-Hernández, E., \& Calmé, S. 2002. Patrones de cacería y conservación de la fauna silvestre en una comunidad maya de Quintana Roo, México. Etnobiología, 2:1-18.

Reid, A. F. 2006. A Field Guide to mammals of North America. Peterson Field Guides. Houghton Mifflin Co. New York. USA. 579 p.

Sáenz-Méndez, J. C., Alfaro-Alvarado, L. D., Carvajal, J. P., \& Carrillo-Jiménez, E. 2005. Una nueva técnica para determinar riqueza y abundancia relativa de aves terrestres: uso de las cámaras-trampa. Zeledonia, 9:22-27.

Santacruz-Salazar, L. J. 2012. Patrón de actividad de Tapirus pinchaque en distintos hábitats y fases lunares, en la hacienda San Antonio, flanco oriental del volcán Tungurahua. Informe de proyecto de trabajo de grado. Universidad Central del Ecuador. $81 \mathrm{p}$.

Santos-Fitá D., E. M. Costa-Neto \& E. J. Cano-Contreras. 2009. El quehacer de la etnozoología. En: Costa-Neto E. M., D. Santos Fita y M. Vargas-Clavijo. (coord.). Manual de etnozoología. Una guía teórico-práctica para investigar la interconexión del ser humano con los animales. Tundra Ediciones, Valencia, España. pp. 23-44.

Soberón, J. \& J. Llorente 1993. The use of species accumulation functions for the prediction of species richness. Conserv. Biol., 7: 480-488.

Vargas-Clavijo, M. 2008. Apropiación de la fauna como patrimonio zoocultural. Boletín Patrimonio Hoy No 2. Ministerio de Cultura. Dirección de Patrimonio. Disponible en URL: http://www.micrositios.us/ mgonzalez/min_cultura2008/index. php?idcategoria=8683. Visitado noviembre 16 de 2008.

Vargas-Clavijo, M. 2009. Patrimonio zoocultural: el mundo animal en las expresiones tradicionales de los pueblos. En: Costa-Neto E. M., D. Santos Fita y M. Vargas-Clavijo. (coord.). Manual de etnozoología. Una guía teórico-práctica para investigar la interconexión del ser humano con los animales. Tundra Ediciones, Valencia, España. pp. 118-141.

Zamorano de Haro, P. 2009. La flora y fauna silvestres en México y su regulación. Procuraduría Agraria. Estudios Agrarios. 40(1):159:167. 